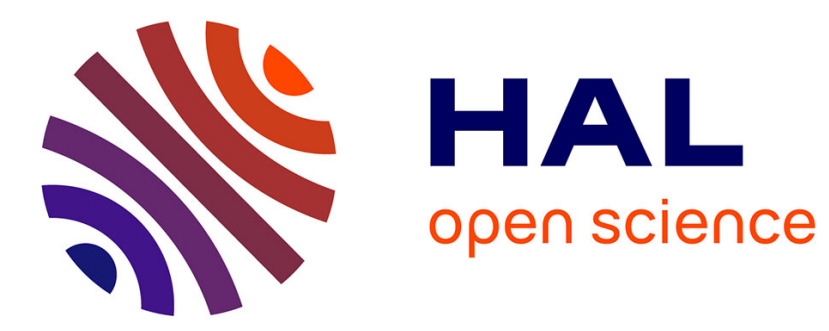

\title{
A Web-Based Platform for Segmentation of Abdominal Organs on CT Images
}

\author{
Xiaoxia Ning, Xuejun Zhang, Qianmei Yang
}

\section{To cite this version:}

Xiaoxia Ning, Xuejun Zhang, Qianmei Yang. A Web-Based Platform for Segmentation of Abdominal Organs on CT Images. 10th International Conference on Intelligent Information Processing (IIP), Oct 2018, Nanning, China. pp.412-420, 10.1007/978-3-030-00828-4_42 . hal-02197778

\section{HAL Id: hal-02197778 \\ https://hal.inria.fr/hal-02197778}

Submitted on 30 Jul 2019

HAL is a multi-disciplinary open access archive for the deposit and dissemination of scientific research documents, whether they are published or not. The documents may come from teaching and research institutions in France or abroad, or from public or private research centers.
L'archive ouverte pluridisciplinaire HAL, est destinée au dépôt et à la diffusion de documents scientifiques de niveau recherche, publiés ou non, émanant des établissements d'enseignement et de recherche français ou étrangers, des laboratoires publics ou privés.

\section{(c)(1)}

Distributed under a Creative Commons Attribution| 4.0 International License 


\title{
A Web-based Platform for Segmentation of Abdominal Organs on CT Images
}

\author{
Xiaoxia Ning $^{1,2}$, Xuejun Zhang ${ }^{1,2} *$,Qianmei Yang ${ }^{1,2}$ \\ ${ }^{1}$ School of Computer, Electronics and Information, Guangxi \\ University, Nanning, Guangxi 530004, P. R. China \\ ${ }^{2}$ Guangxi Key Laboratory of Multimedia Communications \\ and Network Technology, Guangxi University, Nanning \\ 530004, China \\ vivining1110@163.com \\ xjzhang@gxu.edu.cn \\ $812684146 @$ qq. com
}

\begin{abstract}
With the development trend of "Internet Plus", medical staffs hope to change the traditional way of medical diagnosis through the Internet, and medical image processing requires a lot of data, but it is very difficult for hospitals to achieve image data sharing. Therefore, this paper designs a Web-based platform for segmentation of abdominal organs on CT images. Using the software of Xojo and Oray as the development and design of this platform, this paper studies the technology of conversion medical DICOM format image to BMP format, as well as image smoothing, edge detection, image expansion, image corrosion and liver region segmentation. The results of this paper show that the platform realized the image transformation, displaying of medical DICOM format image based on web and the segmentation of CT image of abdominal organs, which is suitable for multiple operating systems, and is convenient for hospital clinical departments to view medical images at any time, and improve the diagnostic speed and accuracy. In addition, a large number of medical image data can be collected through the platform.
\end{abstract}

KEYWORDS: Web , DICOM image, Image segmentation, Xojo

\section{$1 \quad$ Introduction}

With the rapid development of Internet concept and technology, as well as the continuous innovation of data storage technology such as cloud storage and big data, medical image processing technology has developed from the past stand-alone mode to network and digital medical imaging equipment, toward being more functional, comprehensive and intelligent[1]. At the same time, the continuous development of medical equipment has produced a large amount of medical image data, which contains information with great value in diagnosis and treatment. How to use computer and image processing technology to fully excavate medical image information and pro- 
vide strong support for doctors' diagnosis, treatment and surgery has become a problem to be solved[2-4].

Therefore, some commercial companies, the open source community and computer-related researchers are exploring platform-neutral, browser-only visualizations of medical imaging data. Kaspar[5] et al. developed an optimized web-based approach to collaborative stereoscopic visualization, which only requires open source standard Web browsers without the need of client software. Yuan[6] et al. proposed a webbased medical image processing platform, by which authorized users can easily access medical images and powerful computational performance on the workflow server side that they handle using the Internet and innovative database technologies. T.Avudaiappan [7] et al. described the construction of a medical image analysis Web service based on service-oriented architecture (SOA) that can help medical image analyzers, including clinicians and research institutions. Although the above studies have greatly improved medical image processing, there are still some problems remaining to be solved, as follows: (a). Medical image processing needs a large amount of data, but it is difficult for each individual hospital to share picture data[8-9]; (b). The current Internet medical image processing platforms do not support mobile terminals, or only support a single mobile terminal, affect the flexibility of platform use; (c). The development of the existing web-based medical image processing platform is limited to medical image visualization and three-dimensional modeling. (d). Due to the accumulation in medical imaging data, it is getting challenging for radiologists to diagnose lesions in a very short period of time[10]. Therefore, in order to solve these problems, this paper designs a Web-based platform for segmentation of abdominal organs on CT images.

\section{Methods}

\subsection{Web programming platform and virtual server}

Xojo is a cross-platform programming language and multi-platform development tool, which is used as a compiler to develop a CT image segmentation platform for abdominal organs on the basis of its advantages of easy operation. Through Oray dynamic DNS software, the IP and port of the intranet are mapped to the extranet and a domain name is generated. At this time, the extranet can directly access the platform through the domain name to process the abdominal liver CT images.

Fig. 1 shown at below is the overall layout of the interface of the CT image segmentation platform for abdominal organs. Each control is label and can be divided into two parts: control controls and display controls. Among them, label 1-12 are the control widgets, and the function is to upload and display the DICOM format files, read DICOM sequence of each slice of CT images and extract the liver of abdominal organs. Label 13-21 was designed for display control, mainly for image and data display. 


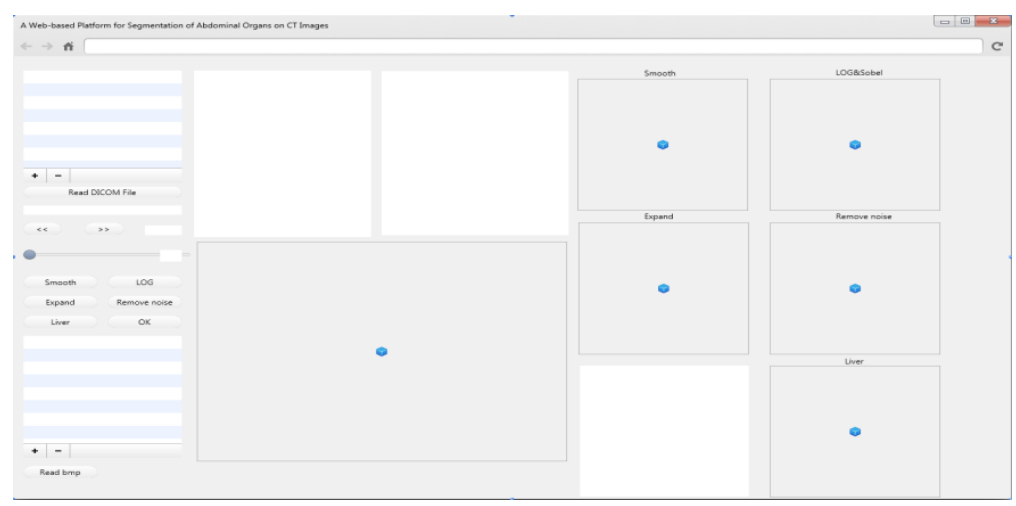

Fig. 1. Interface Layout design

Running Oray, the user's current dynamic IP address will be sent to the dynamic DNS server for interpretation immediately, and the current IP address is tied to a "fixed" domain. Thus, the user's PC can always be accessed through a fixed domain. As shown in Fig.2.

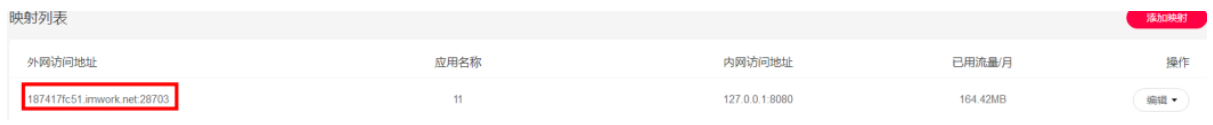

Fig. 2. Intranet mapping

The sequence numbers in Fig. 3 are the remote client's operation procedures. No.1 is for uploading local medical image data, No.2 is for local file storage path, and No. 3 is for DICOM data to be read and processed, then click "OK" to display in the block diagram of No.4, the No.5 red box in the figure is the external network login website.

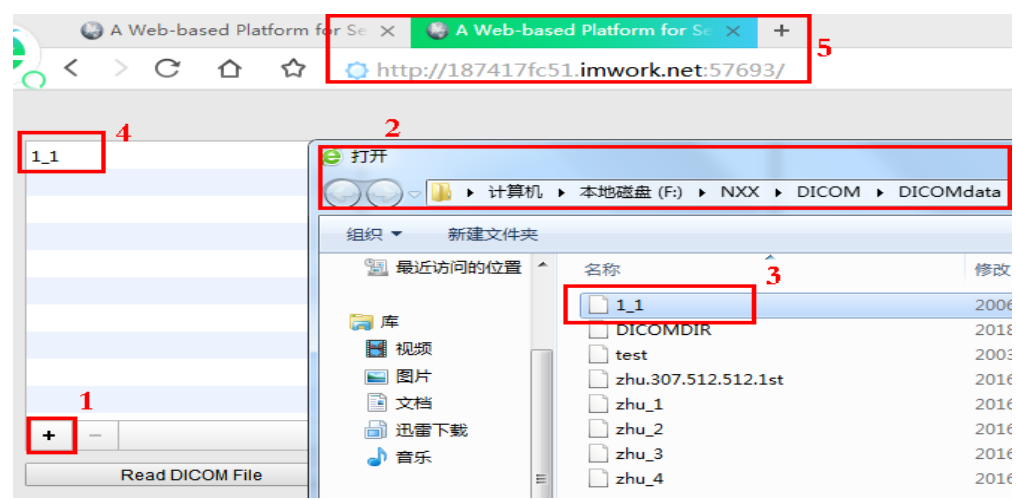

Fig. 3. Platform Operation interface 


\subsection{DICOM file format}

The DICOM file consists of a hypertext header and information objects. The File Meta Information includes identifying information on the encapsulated Data Set. This header consists of a 128 byte File Preamble, followed by a 4 byte DICOM prefix. The four byte DICOM Prefix shall contain the character string "DICOM" encoded as uppercase characters of the ISO 8859 G0 Character Repertoire. This four byte prefix is structured as a DICOM Data Element with a Tag and a Length. The Preamble and Prefix are followed by a set of DICOM Meta Elements with Tags and Lengths. As shown in Fig.4.

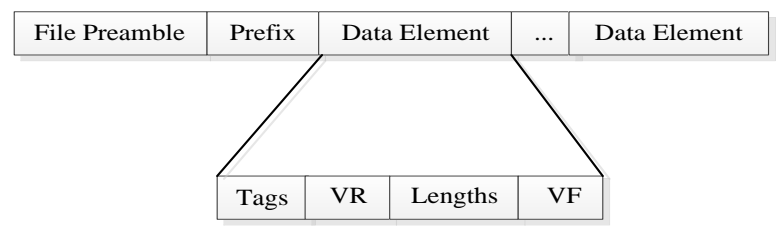

Fig. 4. DICOM format

To convert a DICOM image to common graphic formats (i.e. BMP, JPG, TIF, etc.), we firstly need to read the parameters in the DICOM image file. Through the DICOM specification file or the data dictionary in DICOM standard, query to store the image of the relevant data, mainly includes: Image display Matrix, that is, the image of the width and height; image storage bits, that is, the first pixel occupies a few bytes, if the image is a standard 12-bit grayscale (black and white) image, it will occupy 2 bytes. Find the element labeled with number $(7 \mathrm{fe} 0,0010)$ that indicates the starting position of the image pixel.

\subsection{Segmentation of Abdominal Organ}

The techniques used in liver segmentation include image smoothing, edge detection, expansion and corrosion. This article focuses on edge detection algorithms and arterial vascular localization. The overall processing process is shown in Fig.5.

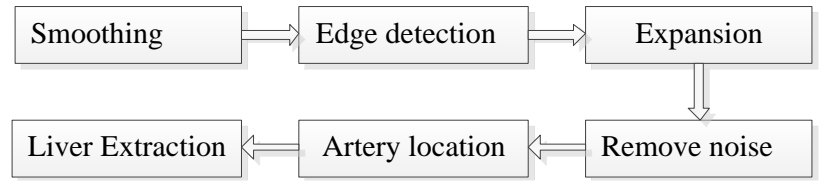

Fig. 5. A schematic workflow of the liver segmentation

\section{A. Edge Detection Algorithm}

The core of traditional edge segmentation methods is edge detection operators, such as Roberts, Sobel, Prewitt, and Gaussian Laplace gradient operators. Edge detection is achieved by weighted summation of pixel values in different directions. Sobel 
operators can smooth noise, provide more accurate edge information, and also produce multiple false edges.

The calculation formula of Sobel operator is:

$$
\begin{aligned}
& f_{x}^{\prime}(x, y)= f(x-1, y+1)+2 f(x, y+1) \\
&+f(x+1, y+1)-f(x-1, y-1) \\
& f_{y}^{\prime}(x, y)= f(x-1, y-1)+2 f(x-1, y) \\
&+f(x-1, y+1)-f(x+1, y+1) \\
& G(f(x, y))=\left|f_{x}^{\prime}(x, y)\right|+\left|f_{y}^{\prime}(x, y)\right|
\end{aligned}
$$

Where $G(f(x, y))$ is the gradient value of the operator, $f_{x}^{\prime}(x, y), f_{y}^{\prime}(x, y)$ is the grayscale partial differential in the horizontal direction and the vertical direction. Let gradient threshold be $T$, if $G(f(x, y))>T$, we can use this point as edge point or vice versa. As long as you adjust a proper threshold $T$, you can get better test results.

LoG operator detects edge by using the feature that the second derivative of gray inflection point equals 0 . The Laplace operator of grayscale image is expressed as:

$$
\nabla^{2} f=\frac{\partial^{2} f}{\partial x^{2}}+\frac{\partial^{2} f}{\partial y^{2}}
$$

After differential, you can get:

$$
\begin{aligned}
\nabla^{2} f= & (f(x+1, y)+f(x-1, y) \\
& +f(x, y+1)+f(x, y-1)-4 f(x, y))
\end{aligned}
$$

B. Liver Segmentation

To segment the liver region from the entire area of the CT/MRI image from the abdominal, we use labeling method to uniquely identify each region in the CT/MRI image of the abdomen and separate it from other regions based on the unique features of the region to be segmented[11]. In order to obtain the liver area exactly, we also need to determine the location of arterial blood vessels. Based on the above characteristics, we can segment the aorta based on the following reference values. a). Area reference volume: In abdominal CT/MRI images, the area of the aorta is relatively small for the liver area, but there are still some small regions in the image after the erosion. If area is considered as the unique parameters, these small points also affect the division of the aorta, which should be regarded as noise and removed. The area parameter we chose was $1.5 \%$. If the area of the noise area is smaller than the selected parameter, its circularity will be changed to zero. b). Reference: We can see from the abdominal CT/MRI image that the area of the liver or liver and fat layer adheres is the largest in the abdominal medical image and according to the relative position of the 
aortic area and the liver area in the human body, the liver area with the largest area is used as a reference. The horizontal position difference between the center point of the aorta area and the center point of the selected reference area should not be greater than $15 \%$ of the overall width of the abdominal image. c). Arterial segmentation based on circularity: In the shape analysis, for the recognition of circular icons, we can calculate its circularity, and use the circularity as the basis for judging circular icons.

Through the above series of methods, the extracted liver will be finally obtained through programming. The process of program extraction is:1). Find the area with the largest section of the liver area.2). Extract the liver to the top of the maximum position.3). After the extraction of the upper half is over, the liver area is extracted in the lower half, and the complete liver area is finally obtained.

\section{Results}

\subsection{Liver Segmentation}

The experimental material of this study is the DICOM data of 307 slices of CT obtained from cooperative Hospital, which were CT images under different scanning periods. First we click the "Read DICOM File" button in the figure below to upload the local DICOM data, and then click on the "Smooth", "LOG", "Expand", "Remove noise" and "Judge circle" in the figure respectively to call the image smoothing, edge processing, expansion, delete noise, and determine the circle function to extract the final liver area. As shown in Fig. 6.

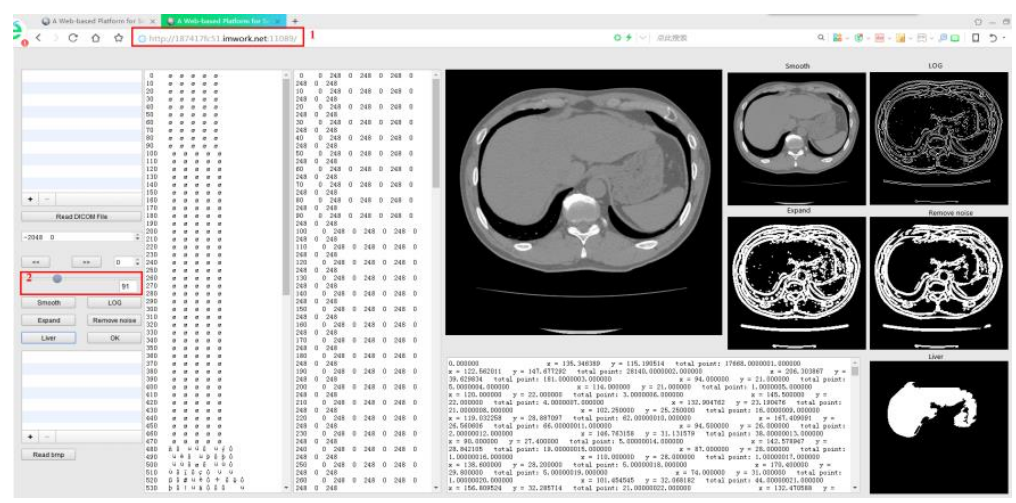

Fig. 6. Abdomen Organ Segmentation

In the figure, the No.1 is the website for remote login of the external network, and the slide bar of the No. 2 can arbitrarily display each CT image. The number of each tablet can be displayed in the box next to it. By dragging the No.1 slide bar in the figure, any one of 307 pieces can be displayed. The following Fig.7 shows the 50th, 100th, 150th, 200th, 250th, and 307th pieces of CT images. 

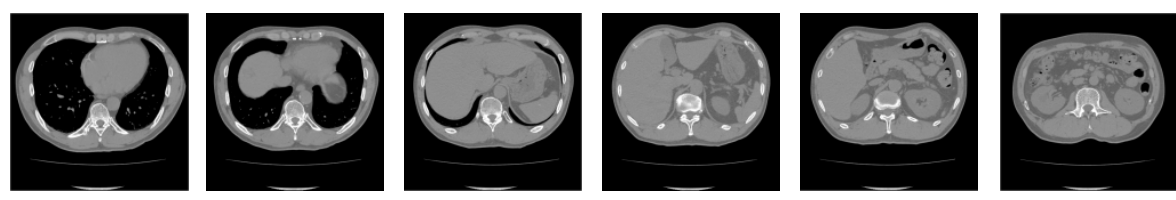

Fig. 7. CT scans at different scan times

The abdominal organ segmentation platform can read 307 abdominal CT images. As long as there are liver regions in each CT image, it can also be correctly extracted. Among them, the liver extraction of any four CT images is shown in the Fig.8.

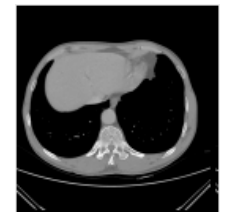

(a)

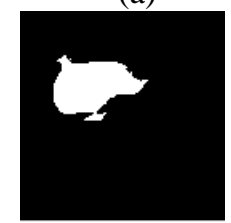

(e)

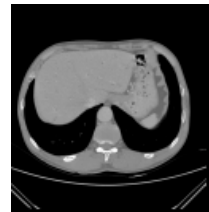

(b)

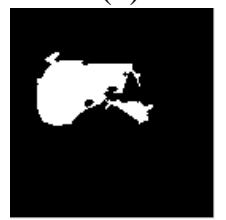

(f)

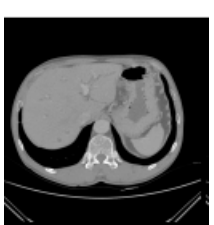

(c)

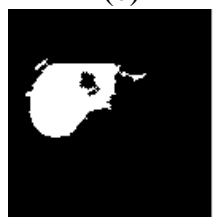

(g)

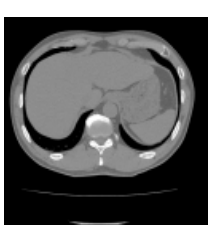

(d)

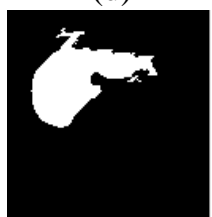

(h)

Fig. 8. The liver extraction of any four CT images

In Fig. 8, (a)-(d) are the original CT images of 65th, 84th, 103th, and 120th, respectively, and (e)-(h) are the liver segmented images corresponding to the top and bottom. It can be seen from the figure that the basic regions of the liver can be correctly segmented, especially (e) (f) (h), but (g) the liver tip portion is not segmented, and the part of the organ and liver that is attached to the liver is not removed.

\subsection{Cross-Platform}

The virtual surgery platform designed in this paper can be accessed and operated on multiple platforms. The tests in the previous chapters were implemented under the windows operating system. The following shows how it works under the Mac operating system. The effect of medical image segmentation under the Mac operating system is shown in Fig. 9 below. Fig. 10 shows the actual operation effect of the Android mobile phone client which uses a bmp format image demo to do the test. 


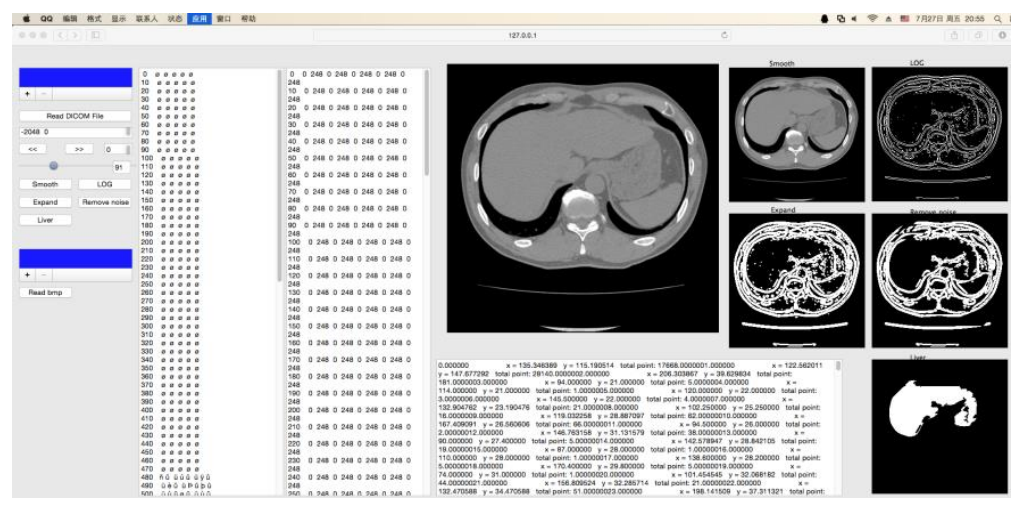

Fig. 9. The effect diagram under the Mac system
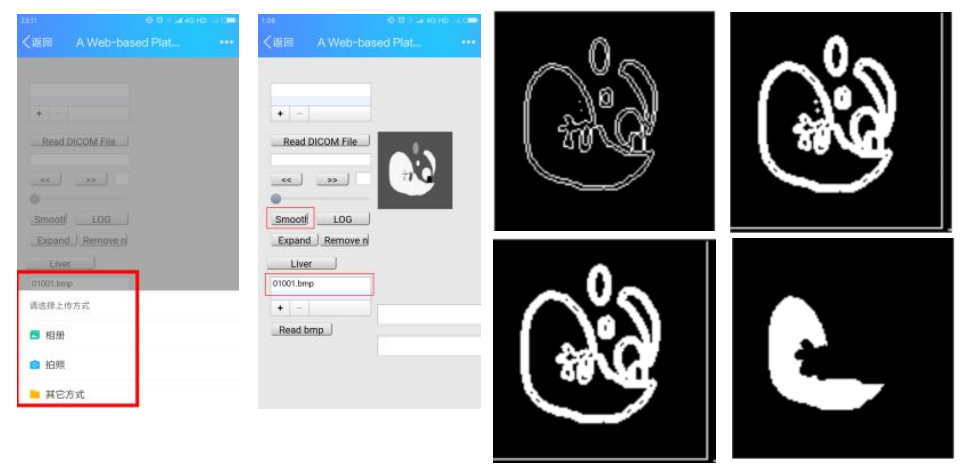

Fig. 10. The effect diagram under the Android system

\section{Conclusions}

The Web-based abdominal organ CT image segmentation platform designed in this paper is equivalent to providing a service. There are mainly the following advantages. a) Economical: It does not need to be equipped with high-end hardware devices. In addition, the client can invoke virtual surgical platform to perform diagnostic reading without installing other plug-ins through the browser. b) Convenience: Based on the Internet+ virtual surgical service platform, users can access the platform whenever and wherever the Internet is available. c) Cross-platform: users can view medical image data on any terminal such as desktop computer, tablet computer, mobile device, etc., which is convenient for hospital clinical departments to view medical images anytime, anywhere, and image segmentation and registration can be performed on the platform to improve diagnosis speed and accuracy. d) Data collection: This software can be used to easily collect data from various hospitals, laying the foundation for future big data, data mining and deep learning. 


\section{Acknowledgments}

This work was supported in part by two research support from the National Natural Science Foundation of China (Nos.81460274 and 81760324), and by JSPS Grant-inAid for Scientific Research on Innovative Areas (grant number 26108005), and in part by Natural Science Foundation of Guangxi (No. 2017JJA170765y).

\section{References}

1. Spiegel M, Hahn DA, Daum V, Wasza J, Hornegger J. "3D graphics on the web: A survey Segmentation of kidneys using a new active shape model generation technique based on non-rigid image registration", vol.1, No.33, pp. 29-39, Computerized Medical Imaging and Graphics, 2009.

2. Mahmoudi SE, Akhondi-Asl A, Rahmani R, Faghih-Roohi, S, Taimouri V, Sabouri A, Soltanian-Zadeh H. "Web-based interactive $2 D / 3 D$ medical image processing and visualization software”, vol.2, No.98, pp.172-182, Computer Methods and Programs in Biomedicine, 2010.

3. Evans A, Romeo M, Bahrehmand A, Agenjo J, Blat J . "3D graphics on the web: A survey”, vol.1, No.41, pp. 43-61, Computers \& Graphics, 2014.

4. Thelen S, Czaplik M, Meisen P, Schilberg D, Jeschke S. "Using off-the-Shelf Medical Devices for Biomedical Signal Monitoring in a Telemedicine System for Emergency Medical Services", vol.1, No.19, pp. 117-123, IEEE Journal of Biomedical and Health Informatics, 2015.

5. Kaspar M, Parsad NM, Silverstein JC. "An optimized web-based approach for collaborative stereoscopic medical visualization”, vol.3, No.20, pp. 535-43, J Am Med Inform Assoc, 2013.

6. Yuan R, Luo M, Sun Z, Shi S, Xiao P, Xie Q. "RayPlus: a Web-Based Platform for Medical Image Processing”, vol.2, No.30, pp. 197-203,Journal of Digital Imaging, 2017.

7. T. Avudaiappan RBNM. "Innovative Framework for Web Based MR Brain Image Segmentation Services for the Medical Image Analyzer", vol.3, No. 6, pp. 465-469, IJCSN - International Journal of Computer Science and Network,2014.

8. Zuojun Z, Dongpo W, Yihe Z, Jinwen D, "Automatic extraction of primary malignant liver tumor for surgical liver resection", Vol.61, No.4, P.17, Journal of Investigative Medicine, 2013.

9. Feng J, Aleksei G, Seungmin R, Zhihong T, YunSheng F, Worku J, Khan A, Shaohui Liu, "Medical image semantic segmentation based on deep learning", vol.5, No.29, pp. 1257-1265, Neural Computing and Applications, 2018.

10. Chiara P, Paul S, Chiara G, Massimo B, Guido B, Marco R. "A tool for validating MRIguided strategies: a digital breathing CT/MRI phantom of the abdominal site", vol.11, No.55, pp. 2001-2014, Medical \& Biological Engineering \& Computing, 2017.

11. Bulat I, Robert K, Bostjan L, Franjo P, Lei X, Toma ` z V. "Segmentation of Pathological Structures by Landmark-Assisted Deformable Models”, vol.7, No.36, pp. 1457-1469, IEEE Transactions on Medical Imaging,2017. 